\title{
AUFSATZ
}

\section{Volksgesetzgebung auf verfassungsrechtlichem Neuland - Rechtsfragen im Zusammenhang mit der baden-württembergischen Volksabstimmung über das „S 21-Kündigungsgesetz“}

\author{
Volker M. Haug
}

Am 27. November 2011 votierte die baden-württembergische Wahlbevölkerung mit 58,9 zu 41,1 Prozent gegen das von ihrer Landesregierung zur Abstimmung gebrachte „Gesetz über die Ausübung von Kündigungsrechten für das Bahnprojekt Stuttgart 21 (S 21-Kündigungsgesetz) “. ${ }^{1}$ Ministerpräsident Winfried Kretschmann, der zuvor für eine gegenteilige Entscheidung geworben hatte, bewertete am Abstimmungsabend die erstmalige Durchführung einer Volksabstimmung im Ländle seit 40 Jahren als einen „großen Sieg für die Demokratie“ und „einen großen Schritt in die Bürgergesellschaft“. ${ }^{2}$ Die Initiative zu einer Volksabstimmung über das Projekt „Stuttgart 21“ war von der SPD ausgegangen, die bereits im Herbst 2010 mit einem entsprechenden parlamentarischen Antrag eine Befriedung des immer heftiger gewordenen Meinungskampfes über das Bahnprojekt angestrebt hatte. ${ }^{3}$ Deshalb warben SPD und Grüne im Landtagswahlkampf auch damit, eine Volksabstimmung herbeizuführen, was dann nach der gewonnenen Wahl entsprechenden Niederschlag in der Koalitionsvereinbarung fand. ${ }^{4}$ Gleichwohl wurde die Volksabstimmung nicht nur politisch heftig kritisiert, sondern auch rechtlich stark angegriffen ${ }^{5}$ - bis hin zu mehreren Anträgen beim Staatsgerichtshof Baden-Württemberg (StGH BW), die Volksabstimmung nicht durchzuführen. ${ }^{6}$ Dies gibt Anlass, die Besonderheiten dieser Volksabstimmung

1 Die Abstimmungsbeteiligung lag bei 48,3 Prozent; zu den Zahlen vgl. http://www.statistik-bw.de/ Wahlen/Volksabstimmung_2011/Land.asp (Abruf am 8. März 2012).

2 Klare Mehrheit für Stuttgart 21, in: Stuttgarter Zeitung vom 28. November 2011, S. 4; vgl. auch die im Vorfeld der Abstimmung an alle Haushalte verteilte Broschüre, http://www.bw-stimmt-ab. de/tl_files/content/5316_Abstimmungsheft_Stuttgart\%2021_Ansicht.pdf (Abruf am 8. März 2012). Gleichwohl kann die Eignung der Volksabstimmung zum S 21-Kündigungsgesetz als Startschuss für mehr Bürgerbeteiligung bezweifelt werden, weil das Verfahren angesichts des im Frühjahr 2010 erfolgten Projektstarts schon (Kosten-)Fakten geschaffen hatte und die Bevölkerung gar nicht mehr wirklich frei entscheiden konnte.

3 Vgl. Antrag vom 8. September 2010, LT-Drs. 14/6896: „Mit einer Volksabstimmung die dringend notwendige breite Akzeptanz der Bürgerinnen und Bürger zur Umgestaltung des Bahnknotens Stuttgart (Stuttgart 21) und zur Neubaustrecke Wendlingen/Ulm zurückgewinnen."

4 Vgl. LT-Drs. 14/6896; Koalitionsvereinbarung zwischen Bündnis 90/Die Grünen und der SPD Baden-Württemberg, Der Wechsel beginnt, S. 29 f.; wegen ihrer Uneinigkeit in der Sache wäre den frischgebackenen Regierungsparteien eine andere Verständigung auch mehr als schwer gefallen.

5 So sprach die Opposition im Landtag von „offenem“ oder „staatlich organisiertem“ Rechtsbruch (MdL Peter Hauk, CDU; MdL Hans-Ulrich Rülke, FDP/DVP), vgl. Plenarprotokoll vom 16. September 2011, S. 456, S. 470 (jeweils rechte Spalte).

6 Besondere Erwähnung verdient in diesem Zusammenhang der Antrag des früheren Rektors der Universität Freiburg Manfred Löwisch vom 7. Oktober 2011, http://www.ivr.uni-stuttgart.de/recht/ 
und des ihr zugrunde liegenden Gesetzentwurfs in rechtlicher Hinsicht näher zu betrachten und anschließend allgemeine partizipationsrechtliche Erwägungen anzustellen.

\section{Zulässigkeit der Volksabstimmung}

\subsection{Erfüllung der Voraussetzungen von Art. 60 LV BW}

Die Volksabstimmung kann in Baden-Württemberg auf zwei Wegen erreicht werden: Zum einen kann das Volk „von unten“ („bottom-up“) eine Volksabstimmung über ein Volksbegehren (Art. 59 Abs. 2, 60 Abs. 1 LV BW) verlangen. Zum anderen kann die Regierung „von oben“ („top down“) eine Volksabstimmung anordnen (Art. 60 Abs. 2, 3 LV BW). Beim „top-down“-Verfahren sind wiederum zwei Varianten zu unterscheiden: Im Fall des Abs. 2 kann die Regierung „ein vom Landtag beschlossenes Gesetz vor seiner Verkündung zur Volksabstimmung bringen, wenn ein Drittel der Mitglieder des Landtags es beantragt", was die Landtagsmehrheit nur mit einer Zweidrittelmehrheit verhindern kann. Im Fall des Abs. 3 ,kann die Regierung eine von ihr eingebrachte, aber vom Landtag abgelehnte Gesetzesvorlage zur Volksabstimmung bringen“, wenn ein Drittel der Abgeordneten dies beantragt. Beide Regelungen geben der Landesregierung eine Option, sich gegen eine politisch andersdenkende parlamentarische Mehrheit durchzusetzen, wenn ein Drittel des Parlaments hinter ihr steht. Während es bei Art. 60 Abs. 2 LV BW um die Verhinderung eines beschlossenen Gesetzes geht (Verhinderungsreferendum), dient Art. 60 Abs. 3 LV BW der Ermöglichung eines vom Parlament abgelehnten Gesetzes (Ermöglichungsreferendum).

Das S 21-Kündigungsgesetz wurde vom (SPD-geführten) Justizministerium unter fachlicher Unterstützung des (grünen) Verkehrsministeriums erarbeitet und dem Ministerrat vorgelegt; dieser fasste nach Durchführung des Anhörungsverfahrens am 12. September 2011 mindestens mit den Stimmen der grünen Kabinettsmitglieder den (Mehrheits-)Beschluss zur Einbringung in den Landtag. ${ }^{7}$ Dieser behandelte den Gesetzentwurf der Regierung am 16. September 2011 in Erster Lesung, anschließend im Ausschuss für Verkehr und Infrastruktur sowie im Ständigen Ausschuss ${ }^{8}$ sowie abschließend in Zweiter Lesung am 28. September 20119; in Übereinstimmung mit den Mehrheitsvoten der beiden Ausschüsse lehnte das Plenum den Gesetzentwurf ab. ${ }^{10}$ Unmittelbar danach rief noch in der Plenarsit-

forschung/materialien/antrag-loewisch.pdf (Abruf am 4. Juni 2012). Auch im Nachgang zur Volksabstimmung sind beim StGH BW 16 fristgerechte Anfechtungsanträge eingegangen, vgl. http://www.lto.de/recht/nachrichten/n/stgh-baden-wuerttemberg-16-verfahren-gegen-volksabstimmung-zum-s-21-kuendigungsgesetz/ (Abruf am 9. März 2012).

7 Zumindest beim Anhörungsentwurf hat auch der der SPD angehörende Justizminister dem Gesetz zugestimmt, vgl. http://www.mvi.baden-wuerttemberg.de/servlet/is/101765/ (Abruf am 8. März 2012); das Einbringungsschreiben von Ministerpräsident Kretschmann datiert vom 12. September 2011, LT-Drs. 15/496, S. 2.

8 Der Ständige Ausschuss gemäß Art. 36 LV BW, $\$ 19$ a GO LT BW hat die Funktion, die Rechte des Parlaments zwischen den Wahlperioden wahrzunehmen; zugleich nimmt er die Funktion des Rechtsausschusses wahr - so auch bei dieser Gesetzesberatung.

9 Die Geschäftsordnung des baden-württembergischen Landtags sieht im Regelfall keine dritte Lesung vor, vgl. $\$ 42$ Abs. 1 GO LT BW.

10 Vgl. Plenarprotokoll 15/13, S. 537, http://www.landtag-bw.de/Wp15/Plp/15_0013_28092011. pdf (Abruf am 31. Mai 2012); nach Internetangaben erfolgte die Ablehnung durch die Abgeord- 
zung die Vorsitzende der Fraktion von Bündnis 90/Die Grünen alle Abgeordneten dazu auf, „nach dieser Sitzung im Foyer den entsprechenden Antrag [auf Durchführung der Volksabstimmung] zu unterschreiben" "11. Der Antrag wurde umgehend von 68 der 138 Abgeordneten unterzeichnet, und noch am selben Tag beschloss die Landesregierung einstimmig, die Volksabstimmung anzuordnen. ${ }^{12}$ Da dieses Vorgehen bereits in der Koalitionsvereinbarung festgelegt worden war, bestritt die Opposition dessen Legitimität; auch von einem „konstruierten Dissens“ war die Rede. ${ }^{13}$ In der Tat handelte es sich um einen „Konflikt mit Ansage“, weil er sich bereits in den Koalitionsverhandlungen abgezeichnet hatte und sowohl die Kabinettsentscheidung als auch die Abstimmungen im Landtag angesichts der politischen Festlegungen der Fraktionen zum Bahnprojekt „Stuttgart 21“ vorhersehbar waren.

Doch verstößt das allein noch nicht gegen Art. 60 LV BW. Entscheidend für die Anwendbarkeit der für ein „top-down“-Verfahren maßgeblichen Verfassungsvorschriften ist vielmehr, ob der institutionelle Konflikt zwischen der Regierung und der Landtagsmehrheit besteht oder nicht. ${ }^{14}$ Und in dieser Hinsicht ist kein Verfassungsverstoß zu erkennen: Die Regierung hat (wenn auch nur mehrheitlich, was aber bei Kollegialorganen rechtlich ohne Belang ist) den Gesetzentwurf beschlossen und dem Landtag zur Beschlussfassung vorgelegt, und der Landtag hat diesen Gesetzentwurf abgelehnt. Bei beiden Beschlüssen gab es kein „taktisches“ Abstimmungsverhalten, wie wir es beispielsweise aus Vertrauensabstimmungen im Vorfeld vorzeitiger Bundestagsauflösungen kennen. Vielmehr haben alle Akteure entsprechend ihren Überzeugungen abgestimmt: Bündnis 90/Die Grünen für das Ausstiegsgesetz, was in der Regierung für eine Mehrheit reichte, und CDU, SPD und FDP/DVP dagegen, was im Landtag zu einer breiten Ablehnungsmehrheit führte. ${ }^{15}$ Damit ist aber der institutionelle Konflikt zwischen Regierung und parlamentarischer Mehrheit - bezogen auf diese eine Sachfrage - weder konstruiert noch vorgetäuscht, sondern tatsächlich existent gewesen. ${ }^{16}$ Man mag es als politisch befremdlich empfinden, dass die zur (generellen) Zusammenarbeit verpflichteten Regierungspartner in diesem Punkt getrennte Wege gegangen sind und die Volksabstimmung (neben der Befriedungsabsicht) zur Lösung ihres internen Konflikts eingesetzt haben ${ }^{17}$; entscheidend ist aber lediglich der institutionelle Konflikt, dessen

neten der CDU, der FDP und - von einzelnen Ausnahmen abgesehen - der SPD, vgl. http://www. kas.de/wf/de/33.29366/ (Abruf am 8. März 2012). Zu den Ausschussvoten siehe LT-Drs. 15/528, S. 1, S. 9.

11 Plenarprotokoll 15/13, S. 540.

12 Vgl. Stephan Eisel, Wer mit Nein stimmt, sagt Ja zu Stuttgart 21. Hintergründe zur Volksabstimmung in Baden-Württemberg, Konrad-Adenauer-Stiftung e.V., Sankt Augustin 2011, http://www. kas.de/wf/de/33.29366/ (Abruf am 8. März 2012).

13 Vgl. zum Beispiel Rudolf Steinberg, Das Volk und die direkte Demokratie, in: FAZ vom 16. Februar 2012, S. 7.

14 So auch (vor einem anderen politischen Hintergrund, weil vor dem Regierungswechsel) KlausPeter Dolde / Winfried Porsch, Gutachterliche Stellungnahme zur Verfassungsmäßigkeit der Initiative der SPD für eine Volksabstimmung über Stuttgart 21 und die Neubaustrecke WendlingenUlm, erstattet im Auftrag der Regierung des Landes Baden-Württemberg vom 1. Oktober 2010, S. 20 ff. mit weiteren Nachweisen, http://www.dhv-speyer.de/kropp/Gutachten_Volksabstimmug_Dolde_u_Porsch.pdf (Abruf am 16. März 2012).

15 Darauf hat Justizminister Rainer Stickelberger im Rahmen der 2. Lesung völlig zutreffend hingewiesen, Plenarprotokoll vom 28. September 2011, S. 536 (rechte Spalte).

16 Vgl. auch LT-Drs. 15/673, S. 4 f.

17 Vgl. Manfred Löwisch, a.a.O. (Fn. 6), S. 13, sieht darin einen Missbrauch des Volksabstimmungsverfahrens. 
vielfältig denkbaren (politischen) Ursachen oder Hintergründe keiner verfassungsrechtlichen Bewertung unterliegen, solange beide Gremien klare (Mehrheits-)Beschlüsse zustande bringen. Auch die „Planmäßigkeit“ dieses Vorgehens ist nicht verfassungswidrig: Der Landtag hätte (etwa aufgrund eines überraschenden politischen Zusammenraufens der Koalitionsparteien) das Ausstiegsgesetz - gegebenenfalls mit Modifikationen - annehmen und damit die Volksabstimmung verhindern können; außerdem sind weder Unvorhergesehenheit noch eine Regierungskrise Voraussetzungen des Art. 60 Abs. 2, 3 LV BW. Allenfalls in einem besonders krassen Fall taktischen Abstimmungsverhaltens könnte man eventuell an einen zur Verfassungswidrigkeit führenden Missbrauch verfassungsrechtlicher Instrumente denken, wofür in diesem Fall jedoch, wie erwähnt, keine Anhaltspunkte vorliegen und was schon angesichts der bundesverfassungsgerichtlichen Billigung der vorzeitigen Bundestagsauflösungen höchst fraglich wäre. ${ }^{18}$

\subsection{Fragestellung}

Gegenstand der Kritik war aber nicht nur das zur Anberaumung der Volksabstimmung führende Verfahren, sondern auch die konkrete Fragestellung. So waren die Abstimmungsberechtigten dazu aufgerufen, dem S 21-Kündigungsgesetz zuzustimmen (mit JA) oder es abzulehnen (mit NEIN). Dies führte zu der als paradox empfundenen Situation, dass die Befürworter des Bahnprojekts mit NEIN und die Projektgegner mit JA stimmen mussten. Wegen der damit verbundenen Gefahr von Missverständnissen wurde der Vorwurf der Intransparenz erhoben. ${ }^{19}$ Zudem kritisierte die CDU-Opposition in diesem Zusammenhang, die Abstimmungsfrage sei tendenziös gestellt und führe den „Abstimmungsbürger hinters Licht“, weil die Frageformulierung das Bestehen von Kündigungsrechten suggeriere; allerdings hätte die von der CDU alternativ vorgeschlagene Fragestellung („Sind Sie für die Kündigung der Verträge zu Stuttgart 21 durch die Landesregierung?" ) das Ja/Nein-Paradoxon ebenso wenig gelöst und ebenfalls das Bestehen eines Kündigungsrechts gedanklich vorausgesetzt. ${ }^{20}$

18 Paul Kirchhof, Gutachtliche Stellungnahme zum Antrag der Fraktion der SPD im Landtag von Baden-Württemberg für eine Volksabstimmung über Stuttgart 21 und die Neubaustrecke Wendlingen-Ulm, erstattet im Auftrag der Regierung des Landes Baden-Württemberg, S. 53 ff., http://www.dhv-speyer.de/kropp/Gutachten_Volksabstimmung_Kirchhof.pdf (Abruf am 16. März 2012), hält die Fälle vorzeitiger Bundestagsauflösungen insofern für nicht vergleichbar. Georg Hermes / Joachim Wieland, Rechtliche Möglichkeiten des Landes Baden-Württemberg, die aus dem Finanzierungsvertrag „Stuttgart 21“ folgenden Verpflichtungen durch Kündigung oder gesetzliche Aufhebung auf der Grundlage eines Volksentscheides zu beseitigen (Gutachten im Auftrag der SPD-Fraktion im Landtag Baden-Württemberg), in: Lars P. Feld / Peter M. Huber / Otmar Jung / Christian Welzel / Fabian Wittreck (Hrsg.), Jahrbuch für direkte Demokratie 2010, Baden-Baden 2011, S. 350 - 393, S. 382 f., betonen in diesem Zusammenhang die rechtliche und tatsächliche Unmöglichkeit, die von einem taktisches Abstimmungsverhalten abweichende „wahre“ Einstellung von Mandatsträgern zu überprüfen.

19 Vgl. Jan Sellner, Verquer - Die Frage zur Abstimmung, in: Stuttgarter Nachrichten online vom 28. September 2011, http://www.stuttgarter-nachrichten.de/inhalt.kommentar-verquer-die-fragezur-abstimmung.40ce02d7-c638-407c-846b-3f1b18bac615.html (Abruf am 9. März 2012).

20 Vgl. CDU: S-21-Volksabstimmung ist tendenziös, in: Schwäbische Zeitung online vom 3. Oktober 2011, http://www.schwaebische.de/region/wir-im-sueden/baden-wuerttemberg_artikel,- 
So berechtigt die Kritik unter sprachlich-kommunikativen Gesichtspunkten sein mag, so zwingend ist die gewählte Frageform aus juristischer Sicht ${ }^{21}$, denn eine Volksabstimmung gemäß Art. 60 Abs. 3 LV BW ist keine politische Volksbefragung, sondern ein Akt der Volksgesetzgebung. Dies schränkt den Spielraum bei der Fragestellung erheblich ein; der Gesetzesbeschluss muss sich - sowohl im parlamentarischen als auch im plebiszitären Fall - direkt und unmittelbar sowie in eindeutiger Weise auf das zu beschließende Gesetz beziehen. Grund dafür ist, dass der Gesetzesbeschluss eine Feststellungsfunktion bezüglich des Gesetzesinhalts erfüllen muss und zugleich den Gesetzesbefehl erteilt. ${ }^{22}$ Daraus folgt zweierlei: Zum einen muss die Frage das betreffende Gesetz nennen (was zu der hier als bürokratisch kritisierten Länge der Frage geführt hat), und zum anderen kann das Gesetz nur mit JA beschlossen und mit NEIN abgelehnt werden. So wäre auch eine Invers-Fragestellung (zum Beispiel „Sind Sie für das Bahnprojekt Stuttgart 21 und lehnen deshalb das S 21-Kündigungsgesetz $a b$ ?") nicht zulässig gewesen. Dasselbe gilt für eine direkte Abstimmung über die Fortführung des Bahnprojekts, der nicht zuletzt die fehlende Landeskompetenz entgegensteht (siehe unten).

\subsection{Ausschlusstatbestände Art. 60 Abs. 6 LV BW}

Schließlich wäre die Volksabstimmung dann unzulässig gewesen, wenn das S 21-Kündigungsgesetz als Abgabengesetz, Besoldungsgesetz oder Staatshaushaltsgesetz im Sinne von Art. 60 Abs. 6 LV BW anzusehen wäre (so genannte Finanztrias). Gegenstand des Gesetzentwurfs ist die Ausübung eines Kündigungsrechts im Rahmen eines multilateralen öffentlich-rechtlichen Vertrags, der unter anderem auch eine Mitfinanzierungspflicht des Landes vorsieht. Da dieses Kündigungsgesetz weder Abgaben noch die Besoldung von Beamten betrifft, scheiden die ersten beiden Varianten offenkundig aus. Allerdings ist es ebenso offenkundig haushaltsrelevant: Wäre das Gesetz beschlossen worden und auf seiner Grundlage eine Kündigung möglich gewesen (dazu unten), hätte sich diese Mitfinanzierungspflicht entweder erledigt oder in eine Schadensersatzpflicht umgewandelt. Doch meint Art. 60 Abs. 6 LV BW nicht eine generelle Haushaltsrelevanz (was sonst nahezu jede gesetzgeberische Maßnahme dem Volksgesetzgeber entzöge und außerdem die Nennung von Abgaben- und Besoldungsgesetzen überflüssig machte $)^{23}$, sondern spricht von „dem“ Staatshaushaltsgesetz. Sowohl der Begriff als auch der

CDU-S-21-Volksabstimmung-ist-tendenzioes-_arid,5141366.html (Abruf am 9. März 2012). Im Übrigen betrifft das Problem, über die Ausübung eines womöglich gar nicht bestehenden Kündigungsrechts abzustimmen, nicht die Zulässigkeit der Fragestellung, sondern das Kündigungsgesetz selbst (siehe unten).

21 Vgl. auch Landesabstimmungsleiterin Christiane Friedrich zitiert nach Florian Buchmaier, Volksentscheid: Frage zu Stuttgart 21 wirft Fragen auf, in: Schwäbische Zeitung online vom 4. November 2011, http://www.schwaebische.de/region/wir-im-sueden/baden-wuerttemberg_artikel,-Volksentscheid-Frage-zu-Stuttgart-21-wirft-Fragen-auf-_arid,5157094.html (Abruf am 9. März 2012).

22 Vgl. Klaus Braun, Kommentar zur Verfassung des Landes Baden-Württemberg, Stuttgart 1984, Art. 59, Rn. 15; Rüdiger Sannwald, in: Bruno Schmidt-Bleibtreu / Hans Hofmann / Axel Hopfauf, Kommentar zum Grundgesetz, 11. Auflage 2008, Art. 77, Rn. 6, Rn. 11; Theodor Maunz, in: ders. / Günter Dürig / Roman Herzog / Rupert Scholz (Hrsg.), Grundgesetz. Kommentar, Loseblattsammlung, München 2011, Art. 77, Rn. 3.

23 So auch Sabine Kropp, „Stuttgart 21“ - Prellbock für den Bundesstaat? Zur Vereinbarkeit von kooperativem Föderalismus und nicht-repräsentativen Formen der Demokratie, in: Europäisches 
bestimmte Artikel machen deutlich, dass damit das Gesetz gemeint ist, das den ein- oder zweijährigen Haushaltsplan mit sämtlichen Einnahmen und Ausgaben des Landes feststellt (79 Abs. 2 LV BW). ${ }^{24}$ Zwar hat das Bundesverfassungsgericht (BVerfG) zum parallelen schleswig-holsteinischen Volksgesetzgebungs-Ausschlusstatbestand entschieden, dass davon alle Gesetze betroffen sind, die „den Haushalt wesentlich beeinflussen“ ${ }^{25}$. Aber Art. 41 Abs. 2 LV SH stellt auf „den Haushalt des Landes“ allgemein und nicht - wie Art. 60 Abs. 6 LV BW - speziell und ausdrücklich (nur) auf „das Staatshaushaltsgesetz“ ab; außerdem wäre unter teleologischen Gesichtspunkten mehr als fraglich, ob der Wegfall einer hohen Ausgabepflicht als „wesentliche“ - das heißt vor allem belastende oder das Gesamtsystem störende - Beeinflussung des Haushalts anzusehen wäre. ${ }^{26}$ Damit ist auch dieser Ausschlusstatbestand des Art. 60 Abs. 6 LV BW für das S 21-Kündigungsgesetz nicht einschlägig.

\subsection{Zwischenergebnis}

Demnach ist festzustellen, dass die Volksabstimmung in Baden-Württemberg am 27. November 2011 als solche zulässig war; insbesondere der dafür erforderliche institutionelle Konflikt war gegeben, und der Haushaltsvorbehalt war nicht einschlägig.

\section{Verfassungsmäßigkeit des S 21-Kündigungsgesetzes}

\subsection{Gesetzgebungskompetenz des Landes?}

Zunächst setzt die Verfassungsmäßigkeit des S 21-Kündigungsgesetzes voraus, dass der Landesgesetzgeber für den Regelungsgegenstand überhaupt inhaltlich zuständig ist. Zwar weist das GG den Ländern in Art. 70 Abs. 1 eine generelle Regelzuständigkeit für die Gesetzge-

Zentrum für Föderalismus-Forschung Tübingen (Hrsg.), Jahrbuch des Föderalismus 2011, BadenBaden 2011, S. 192 - 204, S. 201; Justizminister Rainer Stickelberger, Plenarprotokoll vom 16. September 2011, S. 475; anderer Auffassung BVerfGE, Bd. 102, S. 176, Rn. 92, wonach dadurch die Volksgesetzgebung keineswegs leer laufe: „Vielmehr bleibt der Volksgesetzgebung auch in Fragen von hoher Bedeutung für den Einzelnen und die Allgemeinheit ein Anwendungsbereich. So sind Fragen etwa nach der Reichweite staatlicher Eingriffsbefugnisse auf dem Gebiet der öffentlichen Sicherheit und Ordnung, Fragen der Ausgestaltung der Kommunalverfassung, aber auch des Schulwesens, die weder mittelbar noch unmittelbar wesentliche haushaltswirksame Ausgaben nach sich ziehen, der Volksgesetzgebung zugänglich."

24 Wie hier: Paul Feuchte, in: ders. (Hrsg.), Verfassung des Landes Baden-Württemberg. Kommentar, Stuttgart 1987, Art. 60, Rn. 10; Klaus Braun, a.a.O. (Fn. 22), Art. 59, Rn. 40; Georg Hermes $/$ Joachim Wieland, a.a.O. (Fn. 18), S. 385. Weitgehend dagegen Klaus-Peter Dolde / Winfried Porsch, a.a.O. (Fn. 14), S. 29 ff. mit weiteren Nachweisen, wonach schon wesentliche Auswirkungen auf den Haushalt zum Ausschlussgrund des Staatshaushaltsgesetzes führt. Interessant ist insoweit die Entscheidung des VerfGH Berlin vom 6. Oktober 2009, in: Lars P. Feld / Peter M. Huber / Otmar Jung / Christian Welzel / Fabian Wittreck (Hrsg.), a.a.O. (Fn. 18), S. 322 f., wonach der Haushaltsvorbehalt nur für bereits beschlossene Haushaltsgesetze, nicht aber für künftige Haushalte gelten soll.

25 BVerfGE, Bd. 102, S. 176, S. 185 ff.

26 Vgl. Hans Schneider, Gesetzgebung, Heidelberg 2002, Rn. 181, zum Grund für die Finanzvorbehalte; anderer Auffassung Klaus-Peter Dolde / Winfried Porsch, a.a.O. (Fn. 14), S. 51 f.; Paul Kirchhof, a.a.O. (Fn. 18), S. $36 \mathrm{ff}$. 
bung zu, relativiert diese jedoch erheblich durch die Festlegung umfassender Ausnahmekompetenzen zugunsten des Bundes in den Art. 73 und 74. Danach ist der Bund insbesondere auch für die Gesetzgebung (ausschließlich) zuständig, die „den Verkehr von Eisenbahnen, die ganz oder mehrheitlich im Eigentum des Bundes stehen [...], den Bau, die Unterhaltung und das Betreiben von Schienenwegen der Eisenbahnen des Bundes [...]" betrifft (Art. 73 Abs. 1 Nr. 6a GG). Zu den Schienenwegen als Eisenbahninfrastruktur gehören auch die Bahnhöfe ${ }^{27}$, weshalb das Land Baden-Württemberg keine gesetzliche Regelung über das Bahnprojekt „Stuttgart 21“ treffen kann. Das ist allerdings - wie auch die Finanzierungsvereinbarung als solche - nicht Gegenstand des S 21-Kündigungsgesetzes, das sich auf die Ausübung von (etwaigen) Kündigungsrechten des Landes im Rahmen der multilateralen Finanzierungsvereinbarung, die der Projektfinanzierung und -durchführung zugrunde liegt, beschränkt. Ob das Land an einem Projekt teilnimmt oder sich (soweit rechtlich möglich) daraus zurückzieht, kann denklogisch immer nur eine Entscheidung des Landes sein. An seiner Gesetzgebungskompetenz für das S 21-Kündigungsgesetz besteht danach kein Zweifel. Rechtlich gesehen könnten die übrigen Vertragspartner, insbesondere die Bahn AG, auch im Falle eines Rückzugs des Landes das Projekt fortführen. Auch wenn es sich dabei aus finanziellen und politischen Gründen um eine eher theoretische Option handelt, ist der Regelungsgegenstand des S 21-Kündigungsgesetzes kein Bahnprojekt, sondern ein Handeln des Landes. ${ }^{28}$

\subsection{Unzulässiges Einzelfallgesetz?}

Des Weiteren könnte dem S 21-Kündigungsgesetz entgegen gehalten werden, ein unzulässiges Einzelfallgesetz zu sein. Nach den Grundsätzen der Normenlehre stellen Gesetze - jedenfalls in aller Regel - generell-abstrakte Rechtssätze dar, die unbestimmt viele Fälle für unbestimmt viele Adressaten in der Zukunft regeln. ${ }^{29}$ Dies ist beim S 21-Kündigungsgesetz sicher nicht der Fall, weil es um die Kündigung bestimmter Vereinbarungen geht. Allerdings ist begrifflich das Einzelfallgesetz vom Maßnahmegesetz zu unterscheiden: Während das Einzelfallgesetz im Sinne von Art. 19 Abs. 1 GG eine ganz bestimmte Person betrifft und deshalb ein „Einzelpersonengesetz" darstellt, ist ein Maßnahmegesetz auf einen konkreten Sachverhalt - insbesondere Infrastrukturplanungen - bezogen. ${ }^{30}$ Das S 21-Kündigungsgesetz betrifft zwar vorrangig die (juristische) Person „Bahn AG“, aber eben nicht nur. Rechtlich unmittelbar mitbetroffen sind fraglos auch die anderen Vertragspartner wie die Stadt

27 Vgl. Arnd Uhle, in: Theodor Maunz / Günter Dürig / Roman Herzog / Rupert Scholz (Hrsg.), a.a.O. (Fn. 22), Art. 73, Rn. 145.

28 So im Einzelnen auch Klaus-Peter Dolde / Winfried Porsch, a.a.O. (Fn. 14), S. 55 f.

29 Vgl. Hans Schneider, a.a.O. (Fn. 26), Rn. 35 - 37, betont außerdem die Bedeutung der Allgemeinheit des Gesetzes als maßgeblichen Abgrenzungsfaktor zu Regierungsakten und Urteilen und damit für das Gewaltenteilungsprinzip.

30 Vgl. Barbara Remmert, in: Theodor Maunz / Günter Dürig / Roman Herzog / Rupert Scholz (Hrsg.), a.a.O. (Fn. 22), Art. 19 Abs. 1, Rn. 36; Christoph Degenhart, Staatsrecht I. Staatsorganisationsrecht, München 2010, Rn. 125; Walter Krebs, in: Ingo von Münch / Philip Kunig (Hrsg.), Grundgesetz-Kommentar, München 2012, Art. 19, Rn. 12; Hans Hofmann, in: Bruno Schmidt-Bleibtreu I ders. I Axel Hopfauf, a.a.O. (Fn. 22), Art. 19, Rn. 10; BVerfGE, Bd. 15, S. 126, S. 146 f.; BVerfGE, Bd. 25, S. 371, S. 396. Vgl. auch Hans Schneider, a.a.O. (Fn. 26), Rn. 38 ff., der vom „Individualgesetz" spricht. 
Stuttgart, der Verband Region Stuttgart und die Flughafen Stuttgart GmbH sowie mittelbar die am Bauprojekt beteiligten Unternehmen (die schon einen Zuschlag erhalten haben und die an Ausschreibungsverfahren beteiligt sind). Hinzu kommen die mannigfachen (teilweise auch rechtlichen) Auswirkungen auf die Baustellenanwohner und Bahnkunden. Vor diesem Hintergrund wird deutlich, dass die mit dem Gesetz angestrebte Kündigung der Finanzierungsvereinbarung eine Maßnahme im Rahmen eines Infrastrukturprojekts mit vielfachen Auswirkungen darstellt. Das S 21-Kündigungsgesetz ist demnach ein Maßnahmegesetz, das als solches keinen besonderen verfassungsrechtlichen Anforderungen unterliegt. ${ }^{31}$

Doch selbst wenn man das S 21-Kündigungsgesetz als Einzelfallgesetz ansehen wollte, wäre es nicht ohne weiteres unzulässig, denn das GG geht von einem formellen Gesetzesbegriff aus, der lediglich darauf abstellt, dass ein Gesetz durch die dafür vorgesehenen Organe auf dem dafür vorgesehenen Verfahrensweg beschlossen wird. ${ }^{32}$ Einzelfallgesetze sind nach Art. 19 Abs. 1 Satz 1 GG nur dann ausdrücklich verboten, wenn es um eine förmliche Grundrechtseinschränkung geht. Eine solche liegt hier nicht vor, auch nicht bezüglich des Selbstverwaltungsrechts des Vertragspartners Stadt Stuttgart, da zum einen die Gemeinden nicht grundrechtsfähig sind und zum anderen die Selbstverwaltungsgarantie gemäß Art. 28 Abs. 2 GG kein Grundrecht, sondern eine institutionelle Rechtssubjektsgarantie darstellt. ${ }^{33}$ Auch die Bahn AG ist als 100-Prozent-Tochter des Bundes nicht grundrechtsfähig, da sich der Staat und seine Einrichtungen nicht durch Rechtsformwahlen von ihren Grundrechtsbindungen frei machen können (,keine Flucht ins Privatrecht“). ${ }^{34} \mathrm{Im}$ Übrigen müssen Einzelfallgesetze (nur) dem Gleichbehandlungsgrundsatz des Art. 3 Abs. 1 GG Genüge tun, der das willkürliche - also nicht durch Sachgründe gerechtfertigte - gesetzgeberische Herausgreifen eines Regelungsgegenstandes aus vergleichbaren Fällen verbietet. ${ }^{35}$ Beim S 21-Kündigungsgesetz fehlen zum einen die für die Ungleichbehandlung erforderlichen Parallelfälle, und zum anderen liegen die nötigen sachlichen Gründe bereits in den in der Gesetzesbegründung genannten verkehrsbezogenen und kostenmäßigen Risiken. ${ }^{36}$

31 Vgl. BVerfGE, Bd. 25, S. 371, Ls. 1, S. 396; BVerfGE, Bd. 36, S. 383, S. 400; Hans Hofmann, a.a.O. (Fn. 30), Art. 19, Rn. 10; Friedrich E. Schnapp, in: Ingo von Münch / Philip Kunig (Hrsg.), a.a.O. (Fn. 30), Art. 20, Rn. 45; so auch Georg Hermes / Joachim Wieland, a.a.O. (Fn. 18), S. 377. Ein unzulässiges Maßnahmegesetz hätte nach Paul Kirchhof, a.a.O. (Fn. 18), S. 44 f., aber dann vorgelegen, wenn es unmittelbar den FinV aufzuheben versucht hätte.

32 Vgl. Christoph Degenhart, a.a.O. (Fn. 30), Rn. 126 ff.

33 Vgl. Barbara Remmert, a.a.O. (Rn. 30), Art. 19 Abs. 3, Rn. 46; Wolfgang Löwer, in: Ingo von Münch I Philip Kunig (Hrsg.), a.a.O. (Fn. 30), Art. 28, Rn. 45 f.; siehe aber auch Manfred Löwisch, a.a.O. (Fn. 6), S. 14.

34 Streitig, wie in: BVerwGE, Bd. 113, S. 208, S. 211 und ihm folgend BGH, in: DÖV 2004, S. 439 f.; Horst Dreier, in: ders. (Hrsg.), Grundgesetz. Kommentar, Tübingen 2006, Art. 1 Abs. 3, Rn. 69 und Art. 19 Abs. 3, Rn. 70; Jan Ziekow, Öffentliches Wirtschaftsrecht, München 2010, \$ 7, Rn. 31 f.; Georg Hermes / Joachim Wieland, a.a.O. (Fn. 18), S. 377. Gegenansicht bei Markus Möstl, in: Theodor Maunz / Günter Dürig / Roman Herzog / Rupert Scholz (Hrsg.), a.a.O. (Fn. 22), Art. 87e, Rn. 100 ff., insbesondere S. 102 f.; Robert Uerpmann-Wittzack, in: Ingo von Münch / Philip Kunig (Hrsg.), a.a.O. (Fn. 30), Art. 87e, Rn. 10 und Art. 87 f, Rn. 17; Kay Windthorst, in: Michael Sachs (Hrsg.), Grundgesetz. Kommentar, München 2011, Art. 87e, Rn. 49. Vermittelnder Ansatz bei Kay Ruge, in: Bruno Schmidt-Bleibtreu / Hans Hofmann / Axel Hopfauf, a.a.O. (Fn. 22), Art. 87 e, Rn. 5.

35 Vgl. Hans Hofmann, a.a.O. (Fn. 30), Art. 19, Rn. 8; Hans Schneider, a.a.O. (Fn. 26), Rn. 45 f.; BVerfGE, Bd. 25, S. 371, Ls. 2, S. 398; BVerfGE, Bd. 85, S. 360, S. 374.

36 Vgl. LT-Drs. 15/496, S. 4 ff. 


\subsection{Verletzung des Gewaltenteilungsprinzips?}

Ungewöhnlich am S 21-Kündigungsgesetz ist, dass eine Regierung mit einem von ihr selbst initiierten Gesetzentwurf das Parlament beziehungsweise das Volk auffordert, sie zur Ausübung eines Kündigungsrechtes zu verpflichten. Fraglos könnte die Regierung, falls sie ein Kündigungsrecht hätte, dieses auch ohne gesetzliche Verpflichtung ausüben ${ }^{37}$; schließlich konnte sie (in personell und politisch anderer Besetzung) im Jahr 2009 die Finanzierungsvereinbarung auch ohne gesetzlichen Auftrag abschließen. ${ }^{38}$ Üblicherweise zählen der Abschluss und gegebenenfalls die Kündigung von Finanzierungsvereinbarungen zum klassischen Vollzugsgeschäft politisch getroffener Entscheidungen und sind damit dem exekutiven Handlungsbereich zuzuordnen. Indem hier nun aber der Gesetzgeber (Parlament oder Volk) die Regierung zur Kündigung verpflichten soll, ohne ihr dabei eigene Entscheidungsspielräume wie etwa ein Ermessen einzuräumen, zieht die Legislative diese Vollzugsentscheidung unmittelbar an sich. ${ }^{39}$ Darin könnte eine Verletzung des Gewaltenteilungsprinzips gemäß Art. 20 Abs. 2 Satz 2 GG beziehungsweise Art. 25 Abs. 1 Satz 2 LV BW liegen. Dies wäre dann der Fall, wenn den Gewalten die Aufgaben abstrakte Normsetzung (Legislative) und konkreter Vollzug (Exekutive) konturenscharf und überlappungsfrei zugewiesen wären. Dies ist indes nicht der Fall; vielmehr steht jeder dieser beiden Staatsgewalten - denen die Staatsleitung „zur gesamten Hand“ übertragen ist ${ }^{40}$ - nur ein bestimmter Kernbereich ausschließlicher Kompetenzen zu. Dieser Kernbereich ist im Fall der Legislative relativ breit und umfasst insbesondere die formellgesetzliche Rechtsetzung, alle „wesentlichen“ Entscheidungen im Sinne der Wesentlichkeitstheorie des BVerfG (wozu vor allem das Erfordernis einer Rechtsgrundlage bei Grundrechtseingriffen zählt) und - gestützt auf das Demokratieprinzip - alle wichtigen politischen Entscheidungen wie beispielsweise auf Bundesebene der Einsatz militärischer Streitkräfte. ${ }^{41}$ Die Exekutive verfügt dagegen über einen schmaleren so genannten „Kernbereich exekutiver Eigenverantwortung“, der laufende Entscheidungsprozesse, die regierungsinterne Willensbildung und besondere Einzelentscheidungen wie zum Beispiel im Bund die Aufstellung des Haushaltsplans und in den Ländern die Festlegung des Abstimmungsverhaltens im Bundesrat umfasst. ${ }^{42}$

37 Davon geht auch die Regierung selbst aus („möglicherweise“), vgl. Justizminister Rainer Stickelberger, Plenarprotokoll vom 16. September 2011, S. 476 (linke Spalte).

38 Daran ändert auch der Umstand nichts, dass der Landtag im Nachgang dem FinV zugestimmt hat (LT-Drs. 14/4438); diese Zustimmung dient der Einbindung des Inhabers des Budgetrechts, ist aber entgegen Fabian Wittreck, Demokratische Legitimation von Großvorhaben, in: ZG 2011, S. 209, S. 212 f., kein Fall des Zustimmungserfordernisses gemäß Art. 50 Abs. 2 LV BW; dieses gilt nur für Staatsverträge mit Völkerrechtssubjekten, vgl. Klaus Braun, a.a.O. (Fn. 22), Art. 50, Rn. 23.

39 Vgl. Manfred Löwisch, a.a.O. (Fn. 6), S. 12 f.

40 Christoph Degenhart, a.a.O. (Fn. 30), Rn 674.

41 Vgl. Christoph Degenhart, a.a.O. (Fn. 30), Rn. 32 - 35; vgl. zum Beispiel BVerfGE, Bd. 68, S. 1, S. 109 (nicht zwingend in Gesetzesform, aber "nicht am Bundestag vorbei“); BVerfGE, Bd. 121, S. 135, S. 156 ff. (Awacs).

42 Vgl. Christoph Degenhart, a.a.O. (Fn. 30), Rn 618; das Initiativmonopol wird aus Art. 110 Abs. 3 Satz 1, Art. 113 Abs. 1 Satz 1 GG abgeleitet, Markus Heintzen, in: Ingo von Münch / Philip Kunig (Hrsg.), a.a.O. (Fn. 30), Art. 110, Rn. 38; siehe auch den Zustimmungsvorbehalt der Regierung gegenüber dem Parlament gemäß Art. 82 Abs. 1 LV BW für Ausgabenerhöhungen oder Einnahmeminderungen. 
Zwischen diesen Kernbereichen gibt es viele Aufgaben, die grundsätzlich sowohl von der Legislative als auch von der Exekutive wahrgenommen werden können, beispielsweise Planungsentscheidungen oder die Rechtsetzung der Exekutive auf Verordnungsebene. ${ }^{43}$ Dem widerspricht auch nicht das Urteil des Staatsgerichtshofs Baden-Württemberg (StGH BW) vom 18. März 1986, wonach der Volksgesetzgeber in die dem „Kernbereich der vollziehenden Gewalt“ zugeordneten Vorbehalte für den Ministerpräsidenten und die Gesamtregierung in Art. 49 LV BW (unter anderem Richtlinienkompetenz, Stimmabgabe im Bundesrat ${ }^{44}$ nicht eingreifen darf. ${ }^{45}$ Abgesehen davon, dass der StGH BW dabei weniger Art. 45 LV BW als Art. 49 LV BW im Blick hatte ${ }^{46}$, gehört der die vollziehende Gewalt pauschal der Regierung zuweisende Art. 45 Abs. 1 LV BW nicht zum ausschließlichen exekutiven Kernbereich, sondern stellt eine generelle (Teil-)Funktionsbeschreibung dar. ${ }^{47}$ Auch wenn das BVerfG ausdrücklich festgestellt hat, dass aus dem Demokratieprinzip kein genereller Vorrang für das Parlament (und damit auch des Volkes, soweit es als Gesetzgeber tätig ist) gegenüber den anderen Gewalten ,als ein alle konkrete Kompetenzzuordnungen überspielender Auslegungsgrundsatz “ abgeleitet werden darf, ist unstreitig, dass die gesetzgebende Gewalt (erst recht in Gestalt des Volkes) als „Leitgewalt“ in einer Demokratie anzusehen ist. ${ }^{48}$ Daraus folgt, dass die gesetzgebende Gewalt alle Aufgaben, die nicht kompetenzmäßig ausschließlich der Regierung zugewiesen sind (siehe oben), ohne Verletzung des Gewaltenteilungsprinzips an sich ziehen kann, jedenfalls wenn es dafür sachliche Gründe gibt. ${ }^{49}$ Hierzu zählt auch die Kündigung der S 21-Finanzierungsvereinbarung. Da die politische Bedeutung von „Stuttgart 21“ angesichts der heftigen Auseinandersetzung in der Bevölkerung die eines normalen Infrastrukturprojekts längst hinter sich gelassen hat, ist dieser gesetzgeberische Zugriff auch sachlich hinreichend begründet und der exekutive Kernbereich dadurch nicht verletzt. ${ }^{50}$

43 Vgl. Christoph Degenhart, a.a.O. (Fn. 30), Rn. 271 betont ebenfalls diesen keiner Staatsgewalt klar zugewiesenen Zwischenbereich; zu Planungsentscheidungen vgl. Wolfgang Ewer, Kein Volksentscheid über die Zulassung von Infrastrukturprojekten, in: NJW 2011, S. 1328, S. 1329, der diese dem Volksgesetzgeber wegen fehlender Diskussions- und Veränderungsmöglichkeit verwehren möchte.

44 Dazu kritisch Hans-Wolfgang Arndt, in: VBIBW 1986, S. 416, S. 417, der diese Bereiche - auch unter Verweis auf andere Regelungen in Berlin und Bremen - nicht dem exekutiven Kernbereich zuordnet (also ein parlamentarisches Instruktionsrecht in der Landesverfassung möglich und mit Art. 28 Abs. 1 GG vereinbar wäre).

45 Vgl. StGH BW, in: VBlBW 1986, S. 335 - 337.

46 Auch nach Hans-Wolfgang Arndt, a.a.O. (Fn. 44), S. 416, kommt es bei dieser Entscheidung allein auf Art. 49 LV BW an; Manfred Löwisch, a.a.O. (Fn. 6), S. 11 f., vertritt hier eine stärkere Betonung des Art. 45 LV BW.

47 Detailliert dazu Klaus Braun, a.a.O. (Fn. 22), Art. 45, Rn. 2 ff., insbesondere S. 7 f.; siehe auch Alfred Katz, in: Paul Feuchte (Hrsg.), a.a.O. (Fn. 24), Art. 45, Rn. 16 ff., insbesondere Rn. 20 f.

48 BVerfGE, Bd. 49, S. 89, S. 125 f.; Christoph Degenhart, a.a.O. (Fn. 30), Rn. 266.

49 Diese fordert Christoph Degenhart, a.a.O. (Fn. 30), Rn. 271 wegen des gegenüber dem Gesetzgeber schwächeren Rechtsschutzes vor dem Hintergrund der Rechtsweggarantie des Art. 19 Abs. 4 GG.

50 Anderer Auffassung Paul Kirchhof, a.a.O. (Fn. 18), S. 25, weil die „Exekutivplanung die langfristige Verwirklichung eines Bauvorhabens betrifft, deren Entwicklung in Einzelschritten begleitet, die bautechnische und finanzielle Anpassung an diese Entwicklung vorbereitet, die Planung also eine vorgegebene Grundentscheidung für ein Bauvorhaben gegenwartsgerecht ausgestaltet." 


\subsection{Bestehen eines Kündigungsrechts?}

Der Inhalt des S 21-Kündigungsgesetzes erschöpft sich in der Verpflichtung der Landesregierung, „Kündigungsrechte bei den vertraglichen Vereinbarungen mit finanziellen Verpflichtungen des Landes Baden-Württemberg für das Bahnprojekt Stuttgart 21 auszuüben“. Immer wieder wurde die Frage aufgeworfen, ob ein solches Kündigungsrecht überhaupt besteht. ${ }^{51}$ Der Gesetzestext legt sich dabei mit der Pluralform ohne bestimmten Artikel weder auf ein bestimmtes Kündigungsrecht fest, noch darauf, ob ein solches (bereits) existiert. ${ }^{52}$ Folglich ist der Gesetzestext so zu lesen, dass die Regierung zur Ausübung eines Kündigungsrechts - sofern und sobald es bestehen sollte - verpflichtet sein soll. Dies gibt Anlass, die verschiedenen Ansätze für ein Kündigungsrecht näher zu untersuchen.

(1) Am naheliegendsten wäre es, wenn der „Finanzierungsvertrag zu Stuttgart 21“ vom 2. April $2009(\text { FinV })^{53}$ - um dessen Kündigung es im Wesentlichen geht ${ }^{54}$ - selbst ein Kündigungsrecht enthielte, wie dies bei vielen vertraglichen Regelungen gegeben ist. Doch das Gegenteil ist der Fall: $\$ 15$ Abs. 1 Satz 2 FinV schließt ein ordentliches Kündigungsrecht ausdrücklich aus. Ein vertragliches Kündigungsrecht gibt es also gerade nicht.

(2) Da es sich bei dem FinV um einen öffentlich-rechtlichen Vertrag im Sinne von $\$ \$ 54$ ff. LVwVfG handelt, ergeben sich aus den dafür geltenden gesetzlichen Bestimmungen außervertragliche (und damit unabdingbare) Kündigungsrechte. So sieht $\$ 60$ Abs. 1 Satz 1 LVwVfG ein Kündigungsrecht vor, wenn „Verhältnisse, die für die Festsetzung des Vertragsinhalts maßgebend gewesen sind, sich seit Abschluss des Vertrags so wesentlich geändert [haben], dass einer Vertragspartei das Festhalten an der ursprünglichen vertraglichen Regelung nicht zuzumuten ist“ und „eine Anpassung nicht möglich oder einer Vertragspartei nicht zuzumuten ist“. Dies entspricht der im Vertragsrecht tradierten Figur des Wegfalls der Geschäftsgrundlage. ${ }^{55}$ Auf diesen Kündigungsgrund setzte die Landesregierung offenbar die größten Hoffnungen; jedenfalls hat sie insoweit den größten argumentativen Aufwand betrieben. ${ }^{56}$

(a) Ein Argumentationsstrang bezieht sich dabei auf die Kostenentwicklung beim Bahnprojekt „Stuttgart 21“. Bereits zum Zeitpunkt der Einbringung des Gesetzentwurfs lag die offizielle Kostenschätzung mit 4,088 Milliarden Euro erheblich über der in $\$ 5$ FinV vereinbarten Kostenobergrenze von 3,076 Milliarden Euro. Aus dieser Entwicklung und anderen Indizien leitet die Gesetzesbegründung die Prognose ab, dass auch der zusätzlich vereinbarte Kostendeckel von 4,526 Milliarden Euro nicht gehalten werden könnte. ${ }^{57}$ Doch selbst

51 In beiden befassten Landtagsausschüssen stand diese Frage im Mittelpunkt der Auseinandersetzung, vgl. LT-Drs. 15/528, S. 2 ff.

52 Vgl. LT-Drs. 15/496, S. 12 (Gesetzesbegründung); dazu Justizminister Rainer Stickelberger in der Debatte zur 1. Lesung, Plenarprotokoll vom 16. September 2011, S. 473 (linke Spalte): „Das kann auch ein Kündigungsrecht sein, das heute noch nicht besteht.“; in diesem Sinne auch in LT-Drs. 15/673, S. 3 (zu Frage 2).

53 LT-Drs. $14 / 4382$.

54 LT-Drs. 15/496, S. 13.

55 Vgl. Heinz Joachim Bonk, in: Paul Stelkens / ders. / Michael Sachs (Hrsg.), Verwaltungsverfahrensgesetz. Kommentar, München 2008, \$ 60, Rn. 12.

56 Vgl. Justizminister Rainer Stickelberger bei der Beratung im Ständigen Ausschuss, LT-Drs. 15/528, S. 4.

57 Vgl. LT-Drs. 15/496, S. 15. 
wenn dies zuträfe, kann daraus kein Wegfall der Geschäftsgrundlage abgeleitet werden ${ }^{58}$, denn die Vertragspartner - also auch das Land - gehen im FinV von der Möglichkeit aus, dass es zu Kostensteigerungen kommt. Deshalb haben sie in $\$ 8$ detaillierte Regelungen für den Fall von „Kostenänderungen des Projekts“ getroffen. Diese umfassen zunächst in $\$ 8$ Abs. 3 eine Reihe von den verschiedenen Kostenträgern zugewiesenen Einzelposten bei Überschreitung der 3,076 Milliarden Euro-Grenze; addiert man diese Einzelposten, gelangt man zum Kostendeckel von 4,526 Milliarden Euro. Darüber hinaus regelt $\$ 8$ Abs. 4 FinV, dass „im Falle weiterer Kostensteigerungen“ (also über 4,526 Milliarden Euro hinaus) „die EIU ${ }^{59}$ und das Land Gespräche auf[nehmen]“. Daraus ergibt sich im Umkehrschluss, dass auch die 4,526 Milliarden Euro-Grenze nach dem Willen der Vertragspartner keine absolute Kostengrenze darstellt, sondern in diesem Fall über die Verteilung der weiteren Zusatzkosten zu sprechen sein würde (und nicht über das „Ob“ des Projekts). Wenn aber eine solche Kostensteigerung - die zumal bei großen Infrastrukturprojekten keineswegs ungewöhnlich sein dürfte - von Anfang an im Vertragstext einkalkuliert worden ist, kann sich keine Vertragspartei später deswegen auf einen Wegfall der Geschäftsgrundlage berufen. ${ }^{60}$

(b) Ein anderer Argumentationsstrang der Regierung zur Begründung des Wegfalls der Geschäftsgrundlage stützt sich auf das Demokratieprinzip, wie es von Georg Hermes und Joachim Wieland herausgearbeitet worden ist. Danach soll die „positive politische Gesamteinschätzung des Projektes Stuttgart $21^{\prime \prime}$ als eines der in $\$ 60$ Abs. 1 LVwVfG genannten (von den Vertragsparteien vorausgesetzten) „Verhältnisse“ anzusehen sein, das im Falle eines gegenteiligen Votums im Rahmen einer Volksabstimmung nicht mehr gegeben wäre. Aus dem Demokratieprinzip folge, dass ein „in Gesetzesform gefasster Volkswille, der die bisherige politische Gesamtbewertung zugunsten des Projektes Stuttgart 21 revidieren würde“, dazu führen würde, dass „dem Land Baden-Württemberg ein weiteres Festhalten an dem Finanzierungsvertrag [...] nicht mehr zuzumuten wäre“; denn „das Demokratie- und das Rechtsstaatsprinzip lassen es nicht zu, dass ein von der Landesregierung ausgehandelter und unterzeichneter Vertrag sich gegen ein in Gesetzesform zum Ausdruck gebrachtes Votum des Volkes durchsetzt“. ${ }^{61}$ Zur weiteren Begründung wird darauf verwiesen, dass der Gesetzgeber auch jederzeit frühere Gesetze aufheben könne, was dann wegen des Gesetzesvorrangs gemäß Art. 20 Abs. 3 GG „erst recht “ für öffentlich-rechtliche Verträge gelten müsse. ${ }^{62}$

Hierzu ist zunächst einzuwenden, dass Gesetz und Vertrag ihrem Wesen nach völlig verschiedene Regelungsinstrumente sind. Ein Gesetz gilt in gleicher Weise für alle Rechtsunterworfenen unabhängig von ihrem Willen und wird damit im Subordinationsverhältnis einseitig von oben nach unten „dekretiert“, kann folglich auch ebenso einseitig modifiziert oder aufgehoben werden. Ein Vertrag dagegen dient dazu, auf einer grundsätzlichen Ebene der Gleichrangigkeit im Wege der Freiwilligkeit eine wechselseitige Rechtsbindung zu er-

58 Anderer Auffassung insoweit Rainer Stickelberger bei der Beratung im Ständigen Ausschuss, LTDrs. 15/528, S. 4.

59 Hinter der Abkürzung „EIU“ verbergen sich die verschiedenen Eisenbahninfrastrukturunternehmen, die am FinV beteiligt sind.

60 Nach Christian Grüneberg, in: Otto Palandt, Bürgerliches Gesetzbuch, München 2011, \$313, Rn. 10, schließen sich Vertragstext und Geschäftsgrundlage sogar gegenseitig aus.

61 Georg Hermes / Joachim Wieland, a.a.O. (Fn. 18), S. 372; Justizminister Rainer Stickelberger, LTDrs. 15/528, S. 4 f., betont, dass die Regierung dieses Gutachten für überzeugend halte und ihrer Position zugrunde lege.

62 Georg Hermes / Joachim Wieland, a.a.O. (Fn. 18), S. 372. 
zeugen. Dies gilt nicht nur beim privatrechtlichen, sondern auch beim öffentlich-rechtlichen Vertrag - insbesondere dann, wenn es sich wie hier um keinen so genannten „subordinationsrechtlichen Vertrag“ im Verhältnis Staat/Bürger handelt, dessen Inhalt auch hoheitlicheinseitig als Verwaltungsakt verfügt werden könnte ( $\$ 54$ Satz 1 LVwVfG), sondern um einen „koordinationsrechtlichen Vertrag“ eines Bundeslandes mit unter anderem einem Bundesunternehmen und einer Kommune. ${ }^{63}$ Diese völlige Typenverschiedenheit bringt mit sich, dass kein lineares Rangverhältnis zwischen Gesetz und (öffentlich-rechtlichem) Vertrag besteht. Außerdem betrifft der angesprochene Vorrang des Gesetzes einen völlig anderen Sachverhalt, nämlich die Einschränkung der Handlungsfreiheit der Verwaltung bei Vorliegen einschlägiger Gesetze. ${ }^{64}$ Dies wirkt sich beim öffentlich-rechtlichen Vertrag in Form des Rechtmäßigkeitsgebots gemäß \$\$ 59, 62 LVwVfG, 134 BGB aus. Solange aber ein Vertrag gegen kein (allgemeines) Gesetz verstößt, ist auch der Gesetzesvorrang nicht einschlägig. Insbesondere kann nicht durch Gesetz ein Vertrag gezielt ausgehebelt werden, weil sonst die hoheitliche Vertragspartei ihre Machtmittel missbrauchen würde (was hier aber auch nicht vorliegt, weil das S 21-Kündigungsgesetz ja nicht versucht, den FinV direkt aufzuheben). ${ }^{65}$

Weder die hohe staatsrechtliche Bedeutung des Gesetzes als staatlicher Willens- und Entscheidungsakt noch die besondere Stellung des Volkes als Ausgangspunkt aller staatlichen Gewalt (Art. 20 Abs. 2 GG) ändern etwas daran, dass diese nur die Willensbildung innerhalb des Rechtssubjekts „Land“ betreffen. Eine Unzumutbarkeit, an einem Vertrag festgehalten zu werden, muss sich aber von außerhalb der betroffenen Vertragspartei ergeben; sonst läge es ja stets in der Hand einer Vertragspartei, durch eine veränderte Willensbildung eigener Organe ${ }^{66}$ eine eigene Unzumutbarkeit herbeizuführen. ${ }^{67}$ Dies aber wäre mit dem Rechtscharakter eines Vertrages, der gerade die externe Bindung gegenüber anderen Rechtssubjekten schafft, unvereinbar. Auf die Spitze getrieben würde die dargestellte Argumentation, über ein (volksbeschlossenes) Gesetz zum Wegfall der Geschäftsgrundlage zu gelangen, letztlich dazu führen, dass jede dem Demokratieprinzip unterliegende Körperschaft (Bund, Länder, Kreise, Gemeinden) einfach durch eine neue demokratische Willensbildung externe Rechtsbindungen auflösen könnte; denn legitimationsrechtlich ist ein vom Volk beschlossenes Gesetz nicht höher einzustufen als ein vom Parlament beschlossenes Gesetz oder - auf kommunaler Ebene - ein Gemeinderatsbeschluss. ${ }^{68}$ Im Ergebnis würde dies bedeuten, dass öffentlich-rechtliche Gebietskörperschaften nicht mehr vertraglich zu binden wären, also vertragsunfähig würden. Das aber wäre mit dem aus dem Rechtsstaatsprinzip folgenden Grundsatz „pacta sunt servanda“, der auch für die Vertragsschlusstätigkeit öffentlich-recht-

63 Vgl. Hans-Peter Bull / Veith Mehde, Allgemeines Verwaltungsrecht mit Verwaltungslehre, Heidelberg 2009, Rn. 849.

64 Vgl. Christoph Degenhart, a.a.O. (Fn. 30), Rn. 287, formuliert es noch einfacher: „Vorrang des Gesetzes bedeutet zunächst nichts anderes als Bindung der Verwaltung an das geltende Recht."

65 Vgl. Klaus-Peter Dolde / Winfried Porsch, a.a.O. (Fn. 14), S. 64 f.

66 Auch das Volk wird vom Grundgesetz letztlich „nur“ als ein Organ des Staates angesehen, vgl. Horst Dreier, a.a.O. (Fn. 34), Art. 20, Rn. 86.

67 So auch Klaus-Peter Dolde / Winfried Porsch, a.a.O. (Fn. 14), S. 65, wonach „der öffentlich-rechtliche Vertrag [...] nicht zur willkürlichen Disposition einer Vertragspartei [steht]“. Vgl. in diesem Zusammenhang auch die zivilrechtliche Unbeachtlichkeit des so genannten Motivirrtums.

68 Vgl. Fabian Wittreck, a.a.O. (Fn. 38), S. 222; Hans Schneider, a.a.O. (Fn. 26), Rn. 182; Bernd Grzeszick, in: Theodor Maunz / Günter Dürig / Roman Herzog / Rupert Scholz (Hrsg.), a.a.O. (Fn. 22), Art. 20 Abs. 2, Rn. 111; vgl. auch Horst Dreier, a.a.O. (Fn. 34), Art. 20, Rn. 89. 
licher Körperschaften gilt, offenkundig unvereinbar. Demnach lässt sich auf diesem Weg kein Kündigungsrecht generieren, auch keines mit Entschädigungspflicht seitens der kündigenden Vertragspartei. ${ }^{69}$

Zudem führt die starke Betonung des Volkswillens in der dargestellten Argumentation zu einem Zirkelschluss. Wenn das Kündigungsrecht (nur) über die Volksabstimmung zu erreichen gewesen wäre, hätte das S 21-Kündigungsgesetz seinen Kündigungsgrund erst durch sich selbst geschaffen. Es kann aber nicht Sinn und Funktion eines Gesetzgebungsverfahrens sein, die inhaltlichen Voraussetzungen für die Vollziehbarkeit des betreffenden Gesetzes erst entstehen zu lassen. Insbesondere besteht die Funktion der Volksabstimmung nicht in der Schaffung einer gesteigerten Gesetzeslegitimation, sondern - wie oben dargelegt - in der Auflösung eines institutionellen Konflikts zwischen Parlament und Regierung. Daher steht diesem Argumentationsansatz auch der Einwand des Verfahrensmissbrauchs entgegen.

(3) Des Weiteren kann „die Behörde“ (hier: das Land) gemäß $\$ 60$ Abs. 1 Satz 2 LVwVfG eine Kündigung aussprechen, „um schwere Nachteile für das Gemeinwohl zu verhüten oder zu beseitigen“. Da es sich hierbei um ein Sonderkündigungsrecht der öffentlichen Hand handelt, sind die Anforderungen hoch: Nur wenn „besondere, erhebliche, überragende Interessen der Allgemeinheit die Auflösung des Vertrages gebieten, durch die dem Staat [...] unzumutbare Lasten auferlegt würden“, kommt dieser Weg in Betracht; eine bloße Nachteiligkeit für die öffentliche Hand - wie sie in der Begründung des Gesetzentwurfs ausgeführt wird - reicht dagegen nicht aus. ${ }^{70}$ Das Bahnprojekt „Stuttgart 21“ stellt ein politisch umstrittenes Infrastrukturprojekt dar, das neben zahlreichen Gegnern auch viele Befürworter sowohl im Landtag als auch in der Landesregierung und folglich seine Pro-Argumente hat; die Projektgegner halten den Bahnhofsneubau für unwirtschaftlich, überteuert und verkehrlich nachteilig, aber nicht für eine schwere Bedrohung des Gemeinwohls. Deshalb hat auch die Landesregierung, soweit ersichtlich, diesen Kündigungsgrund nicht ins Spiel gebracht.

(4) Auch aus der behaupteten Verfassungswidrigkeit des FinV wegen unzulässiger Mischfinanzierung lässt sich kein Kündigungsrecht herleiten. Hans Meyer hat dargelegt, dass die Mitfinanzierung des Landes beim Bahnprojekt „Stuttgart 21“ wegen fehlender Verwaltungsund Finanzierungskompetenz in Bahnangelegenheiten gegen den Trennungsgrundsatz des Art. 104a GG verstoße. ${ }^{71}$ Klaus-Peter Dolde und Winfried Porsch wenden dagegen ein, dass mit dem Bahnprojekt zugleich andere Aufgaben erfüllt werden, die in die Zuständigkeit des Landes und der Stadt Stuttgart fallen und daher die Mitfinanzierung von Land und Stadt rechtfertigen. ${ }^{72}$ Noch weitergehender legt Hubertus Gersdorf mit beachtenswerter Argumen-

69 Davon gehen Georg Hermes / Joachim Wieland, a.a.O. (Fn. 18), S. 373, aus; allerdings liegt im Fall einer berechtigten Kündigung gerade kein vertragswidriges, eine Entschädigungspflicht auslösendes Handeln vor, so dass nur in besonderen Ausnahmefällen von Äquivalenzstörungen eine solche Pflicht bejaht werden kann. Vgl. Heinz Joachim Bonk, a.a.O. (Fn. 55), \$ 60, Rn. 25e; Justizminister Rainer Stickelberger, in: LT-Drs. 15/673, S. 3 (zu Frage 3) und in LT-Drs. 15/616, S. 10 ff. (zu Frage 8).

70 Vgl. Heinz Joachim Bonk, a.a.O. (Fn. 55), \$ 60, Rn. 28.

71 So Hans Meyer, Der Stuttgarter Bahnkonflikt aus der Sicht der Finanzverfassung, in: DVBl. 2011, S. 449 ff.; dieser Beitrag geht auf ein entsprechendes Gutachten im Auftrag der Fraktion Bündnis 90/Die Grünen im Landtag Baden-Württemberg zurück.

72 Vgl. Klaus-Peter Dolde / Winfried Porsch, Eisenbahninfrastruktur und Finanzverfassung, dargestellt 
tation dar, dass das für Verwaltungsaufgaben geltende Trennungsgebot des Art. 104a Abs. 1 GG auf die von Verfassungs wegen unternehmerische Tätigkeit der Eisenbahninfrastrukturunternehmen überhaupt nicht anwendbar ist. ${ }^{73}$ Für die hier interessierende Frage, ob ein Kündigungsrecht besteht, hilft die Auseinandersetzung allerdings nicht weiter. Wäre nämlich der FinV wegen unzulässiger Mischfinanzierung verfassungswidrig, wäre dieser öffentlichrechtliche Vertrag automatisch gemäß $\$ \$ 59$ Abs. 2 LVwVfG, 62 LVwVfG i.V.m. $\$ 134$ BGB nichtig ${ }^{74}$ und damit auch nicht mehr kündbar. Wollte man diesen Weg beschreiten, dürfte man den Vertrag nicht kündigen, sondern müsste - gegebenenfalls mit gerichtlicher Hilfe - dessen Nichtigkeit feststellen lassen. Die im Gesetzentwurf genannte Kündigung kann als einseitiges Gestaltungsrecht jedoch nicht in das prozessuale Instrument einer verwaltungsgerichtlichen Feststellungsklage gemäß $\$ 43$ Abs. 1 VwGO auf Feststellung einer solchen Nichtigkeit umgedeutet werden.

(5) Schließlich lassen sich als weitere außervertragliche Kündigungsgründe das außerordentliche Kündigungsrecht aus wichtigem Grund oder aus allgemeinem Leistungsstörungsrecht ( $\$ 62$ Satz 2 LVwVfG in Verbindung mit den Regelungen des BGB) denken. ${ }^{75}$ Ersteres würde eine schwere Pflichtverletzung der Bahn gegenüber dem Land bei der Vertragserfüllung voraussetzen, wofür keine Anhaltspunkte ersichtlich sind. Das allgemeine Leistungsstörungsrecht sieht in den $\$ \$ 323 \mathrm{ff}$. BGB zwar ein Rücktrittsrecht vor (etwa wegen nicht oder nicht vertragsgemäß erbrachter Leistung im synallagmatischen Austauschverhältnis). Doch ist die Anwendbarkeit dieser Regelungen auf die hier vorliegende Vertragskonstellation höchst fraglich, da insoweit die Unterschiede zwischen dem privat- und öffentlichrechtlichen Vertrag, das fehlende Austauschverhältnis und der Charakter einer gemeinsamen Projektverwirklichung zum Tragen kommen. Außerdem sind keine Anhaltspunkte für das Vorliegen der tatsächlichen Voraussetzungen (zum Beispiel Vertragsnichterfüllung) ersichtlich. Demnach eröffnen auch diese theoretisch denkbaren Ansätze im Fall des FinV zu „Stuttgart 21“ keine realistische Perspektive für ein Kündigungsrecht des Landes.

\subsection{Folge des fehlenden Kündigungsrechts: Ausreichender normativer Gehalt?}

Das S 21-Kündigungsgesetz enthält zwei Aussagen: zum einen, dass das Land sich aus dem Bahnprojekt S 21 zurückziehen soll (Ziel), und zum anderen, dass dies durch eine von der Landesregierung auszusprechende Kündigung erfolgen soll (Mittel). Wenn jedoch, wie dargelegt, ein Kündigungsrecht weder besteht noch mit einer gewissen Wahrscheinlichkeit entstehen wird, kann das Mittel nicht eingesetzt und damit das Ziel - schon aus Rechtsgründen! - nicht erreicht werden. Deshalb stellt sich hier die Frage, ob das S 21-Kündigungsgesetz überhaupt einen hinreichenden normativen Gehalt aufweist. Wäre dies zu verneinen, wäre eine Zielvorstellung in Gesetzesform gebracht worden, die gar kein Gesetz ist oder sein kann.

am Beispiel des Bahnprojekts Stuttgart-Ulm, in: NVwZ 2011, S. 833 ff.; die beiden Autoren hatten die (frühere) Landesregierung im Zusammenhang mit dem Abschluss des FinV beraten.

73 Vgl. Hubertus Gersdorf, Kofinanzierung von Eisenbahninfrastrukturprojekten am Beispiel von Stuttgart 21, in: ZG 2011, S. 248, insbesondere S. $260 \mathrm{ff}$.

74 Näher ausgeführt bei Hans Meyer, a.a.O. (Fn. 71), S. 456 f.

75 Vgl. die Ausführungen von Justizminister Rainer Stickelberger (SPD) am 21. September $2011 \mathrm{im}$ Ständigen Ausschuss, LT-Drs. 15/528, S. 4. 
Dann läge ein Missbrauch des Formentyps des Gesetzes vor, was gegen das Rechtsstaatsprinzip verstieße und damit verfassungswidrig wäre. ${ }^{76}$

Allerdings ist für den Formentyp des Gesetzes als Rechtsnorm ein weites Begriffsverständnis zugrunde zu legen: Der Begriff der Rechtsnorm umfasst nicht nur „Rechtsregeln, also Aussagen über die Rechtsfolgen für eine unbestimmte Vielzahl von Fällen, sondern auch die Festlegung von Grundentscheidungen "77. Letztere können beispielsweise Festlegungen eines Rechtsstatus (zum Beispiel Festlegung der Bundeshauptstadt), einer Planung oder eines Programms darstellen; aber in jedem Fall muss die Festlegung als solche verbindlich - also mit rechtlicher Wirkungskraft ausgestattet - sein. In diesem Sinne muss auch das so genannte formelle Gesetz einen normativen Kern enthalten; das dafür stets als Regelbeispiel angeführte Haushaltsgesetz enthält weitreichende verbindliche Festlegungen im staatlichen Innenverhältnis, auch wenn der außerhalb der hoheitlichen Sphäre stehende Bürger weder dadurch gebunden ist, noch sich darauf subjektiv-rechtlich berufen kann. ${ }^{78}$

Dieser normative Kern würde beim S 21-Kündigungsgesetz fehlen, wenn unter keinem vernünftigen Gesichtspunkt ein Kündigungsrecht bestehen oder entstehen könnte. So weit wird man hier indes nicht gehen können: Auch wenn dafür bislang keine tatsächlichen Anhaltspunkte ersichtlich sind, kann sich theoretisch entweder aus schweren Pflichtverletzungen der Bahn gegenüber dem Land, dem Leistungsstörungsrecht (siehe oben) oder aus unüberwindlichen technischen Realisierungshindernissen ${ }^{79}$ ein Kündigungsrecht für das Land ergeben. In diesem Fall könnte der normative Kern daher in der „Platzhalter“- oder „Reserve“-Funktion für den unwahrscheinlichen Fall des irgendwann erfolgenden Eintritts eines Kündigungsgrundes gesehen werden. Ob allerdings diese theoretische Option wirklich als ausreichende normative Substanz eines Gesetzes anzuerkennen ist, kann auf der Basis der - soweit ersichtlich - bisher vorhandenen Literatur und Rechtsprechung nicht zuverlässig geklärt werden. Gegen die Anerkennung des Gesetzescharakters spricht in diesem Fall die extrem geringe Wahrscheinlichkeit des Entstehens eines Kündigungsrechts, was bereits im Gesetzgebungsverfahren abzusehen war (und weshalb die Landesregierung fast ausschließlich mit dem oben widerlegten Wegfall der Geschäftsgrundlage gemäß $\$ 60$ LVwVfG argumentiert hat). Für die Bejahung eines ausreichenden normativen Gehalts kann dagegen angeführt werden, dass dem parlamentarischen wie plebiszitären Gesetzgeber aufgrund seiner hohen demokratischen Legitimation nach allgemeiner Ansicht ein weiter Prognose- und Einschätzungsspielraum zusteht ${ }^{80}$ und davon auch eine sehr theoretische Wirkungsperspektive erfasst sein könnte. In einer Gesamtbewertung ist demnach festzustellen, dass das $S$ 21-Kündigungsgesetz mindestens erheblichen verfassungsrechtlichen Bedenken begegnet. Es ist daher sehr fraglich, ob es einer konkreten verfassungsgerichtlichen Prüfung hätte Stand halten können.

76 Vgl. Michael Sachs, in: ders. (Hrsg.), a.a.O. (Fn. 34), Art. 20, Rn. 89, Rn. 100, Rn. 106; Christoph Degenhart (Fn. 30), Rn. 137; Bernd Grzeszick (Fn. 68), Art. 20 VI, Rn. 36 ff.

77 Hans Schneider, a.a.O. (Fn. 26), Rn. 16.

78 Ausführlich Hans Schneider, a.a.O. (Fn. 26), Rn. 16 ff., insbesondere Rn. 25 f.; von der Wirkungsnotwendigkeit geht auch Thorsten Franz, Der Begriff des Gesetzes - Geschichte, Typologie und neuer Gesetzesbegriff, in: ZG 2008, S. 140, S. 155 f., S. 158 f., aus.

79 Vgl. den Hinweis von Justizminister Rainer Stickelberger im Ständigen Ausschuss, in: LT-Drs. $15 / 528$, S. 4.

80 Vgl. BVerfGE, Bd. 111, S. 333, S. 356; Bernd Grzeszick, a.a.O. (Fn. 68), Art. 20 VI, Rn. 12 - 15. 


\section{Allgemeine partizipationsrechtliche Erkenntnisse}

Die baden-württembergische Volksabstimmung vom 27. November 2011 gibt über die konkret tangierten Rechtsfragen hinaus Anlass, über eine Weiterentwicklung der (verfassungsrechtlichen) Vorschriften über sachunmittelbare oder direkte Demokratie nachzudenken. Dies gilt besonders für den angemessenen und sinnvollen Einsatz von Quoren und für den (Individual-)Rechtsschutz.

\subsection{Einsatz von Quoren}

Die rechtspolitische Diskussion über die Regularien zur baden-württembergischen Volksabstimmung hat sich sowohl im Vorfeld als auch im Nachgang auf die Frage konzentriert, ob das Zustimmungsquorum in Art. 60 Abs. 5 Satz 2 LV BW der Höhe nach angemessen ist. Nach dieser Bestimmung ist ein Gesetzentwurf nur dann durch das Volk wirksam beschlossen, wenn er nicht nur eine Mehrheit in der Abstimmung findet, sondern darüber hinaus die Zustimmung von mindestens einem Drittel aller Abstimmungsberechtigten. BadenWürttemberg liegt mit diesem Quorum im oberen Bereich, aber auch viele andere Länder sind dem so genannten „Kieler Modell“ gefolgt und haben hohe Zustimmungsquoren von 20 bis 33 Prozent festgelegt; einsam an der Spitze liegt das Saarland mit einem „in der Praxis unerreichbaren“ Zustimmungsquorum von 50 Prozent (Art. 100 Abs. 3 LV SL). ${ }^{81}$

Der offenkundige Grund für Quoren liegt darin, zu verhindern, dass kleine, gut organisierte, aber nicht repräsentative Minderheiten an einem weitgehend passiven Volk vorbei Gesetzesbeschlüsse über Volksbegehren und -entscheid herbeiführen können. ${ }^{82}$ Doch ob dieses berechtigte gesetzgeberische Ziel über hohe Zustimmungsquoren sinnvoll umzusetzen ist, darf bezweifelt werden. Denn dadurch ist ein zentrales Argument für direktdemokratische Instrumente erheblich gefährdet, nämlich die Akzeptanz. Trifft das Volk als letzte und höchste Instanz in einer Demokratie eine Entscheidung, wird dies - sogar bei einem hoch emotionalisierten Konflikt wie „Stuttgart 21“ - von der ganz überwältigenden Mehrheit der Bevölkerung als (gegebenenfalls politisch unliebsame, aber so getroffene) Klärung der Streitfrage akzeptiert. ${ }^{83}$ Tritt aber der Fall auf, dass das Volk mit einer Mehrheit entscheidet, die das (hohe) Zustimmungsquorum nicht erreicht, fühlt sich die Mehrheit um ihren Abstimmungssieg betrogen und (jedenfalls in weiten Teilen) nicht zur Akzeptanz verpflichtet. Dies war auch in Baden-Württemberg im Vorfeld der Volksabstimmung zu beobachten, wo maßgebliche Politiker der Grünen im Gegensatz zu ihrem Ministerpräsidenten wenig Bereit-

81 Vgl. Frank Decker, Zwischen Placebo und Erfolgsmodell. Direkte Demokratie auf Landesebene, in: ZParl, 41. Jg. (2010), H. 3, S. $564-579$, S. 568.

82 Vgl. Klaus Braun, a.a.O. (Fn. 22), Art. 60, Rn. 10.

83 So zum Beispiel beim bayerischen Rauchverbot, das zuvor in zwei gesetzlichen Fassungen umstritten geblieben war. Und obwohl man der heute geltenden, volksbeschlossenen Regelung keinen ausgesprochenen Kompromisscharakter attestieren kann, ist sie vergleichsweise unstreitig. Genau diese Akzeptanzfunktion hat auch die SPD-Fraktion im baden-württembergischen Landtag bei ihrem ursprünglichen Antrag auf Durchführung einer Volksabstimmung in der Antragsüberschrift als Begründung herangezogen (siehe oben in Fußnote 3); ebenfalls diese Akzeptanz- und Befriedungsfunktion betont Fabian Wittreck, a.a.O. (Fn. 38), S. 223; kritisch zur Befriedungsfunktion unter Hinweis auf die Historie Paul Kirchhof, a.a.O. (Fn. 18), S. 11. 
schaft zeigten, das Scheitern des Ausstiegs bei einer unterhalb des Quorums liegenden Mehrheit für das S 21-Kündigungsgesetz zu akzeptieren. ${ }^{84}$ Es sei daher die Hypothese erlaubt, dass im Fall einer Volksabstimmungsmehrheit für den Ausstieg aus dem Bahnprojekt „Stuttgart 21", die das Quorum nicht erreicht hätte, der teilweise gewalttätige Protest gegen das Projekt nicht zurückgegangen wäre, sondern eher noch zugenommen hätte. Die Kritik der „Wutbürger" hätte sich dann nicht nur auf das kritisierte Bahnprojekt und seine Vorgeschichte gerichtet, sondern auch eine „Missachtung des Volkswillens“ beklagt. Dadurch hätte sich die Befriedungs- und Akzeptanzfunktion von Volksabstimmungen in ihr Gegenteil verkehrt. Dies aber kann nicht Sinn oder absehbare Folge eines verfassungsrechtlichen Entscheidungsinstruments sein.

Weil es aber auch nicht nur darum gehen kann, der Abstimmungsmehrheit ihren Sieg zu „gönnen“, sondern auch sichergestellt sein muss, dass die Volksgesetzgebung einen gewissen Grad an Repräsentativität erreicht, sind Hürden zweifellos erforderlich. Allerdings erscheinen „Zugangshürden“ wesentlich geeigneter als „Zustimmungshürden“, um die Akzeptanzfunktion mit der Repräsentativitätsfunktion zu verbinden. ${ }^{85}$ Auch über Zugangshürden lässt sich nämlich sicherstellen, dass nur Belange mit hinreichender politischer Bedeutung dem Volk zur Entscheidung vorgelegt werden. Zu solchen Zugangshürden gehört insbesondere das Quorum für ein erfolgreiches Volksbegehren, mit dem die Anzahl von Volksabstimmungen äußerst wirksam gesteuert werden kann. Derzeit variieren diese Quoren zwischen fünf Prozent (Schleswig-Holstein, Hamburg) und 20 Prozent (Hessen, Saarland). ${ }^{86}$ Weitere wichtige Zugangshürden stellen das Vorhandensein und die Länge einer Sammlungsfrist dar. ${ }^{87}$ Dies ist nicht zuletzt in Verbindung mit dem Quorum ein probater Bedeutungsfilter: Lässt sich eine große Anzahl von Unterschriften in kurzer Zeit sammeln, ist eine ausreichende politische Bedeutung wesentlich eher indiziert als bei ebenso vielen Unterschriften, die in einem sehr langen Zeitraum gesammelt wurden. Als weitere Zugangshürden kommen die Regelung über die Sammlungsorte (nur amtliche Auslegung oder freie Sammlung) ${ }^{88}$ und inhaltliche Anforderungen, zum Beispiel ein Kostendeckungsvorschlag, in Betracht. Auch eine obligatorische Prüfung durch das jeweils zuständige Verfassungsgericht (nach Erfüllung aller formalen Voraussetzungen) wäre denkbar und sinnvoll, um zu vermeiden, dass das Volk zur Abstimmung über einen verfassungswidrigen Entwurf aufgerufen wird. In einigen Ländern existieren solche Verfahren zumindest schon als Rechtsschutz der Initianten im Ablehnungsfall, aber auch als Anrufungsmöglichkeit für parlamentarische Minderheiten oder die

84 Vgl. Boris Palmer im Interview mit Michael König, „Das Wunder von Kretschmann kann gelingen“, in: SZ online vom 25. November 2011, http://www.sueddeutsche.de/politik/boris-palmer-zur-svolksabstimmung-das-wunder-von-kretschmann-kann-gelingen-1.1218230 (Abruf am 15. März 2012).

85 So bereits in Bayern, Hessen, Sachsen und teilweise Nordrhein-Westfalen, vgl. Frank Decker, a.a.O. (Fn. 81), S. 568.

86 Vgl. Art. 42 Abs. 1 Satz 5 LV SH; Art. 50 Abs. 2 Satz 8 LV HH; Art. 124 Abs. 1 Satz 1 LV HE; Art. 99 Abs. 2 Satz 3 LV SL.

87 Ausführlich zur praktisch-machtpolitischen Bedeutung der Quoren- und Fristenregelung beim Volksbegehren Frank Meerkamp, Die Bedeutung von Eintragungsfristen beim Volksbegehren im Rahmen der Volksgesetzgebungsverfahren in den deutschen Ländern, in: ZParl, 41. Jg. (2010), H. 3, S. 579 - 598, S. 581.

88 Interessant ist insoweit die Regelung in Thüringen, wo die Initianten des Volksbegehrens zwischen den Sammlungsformen mit unterschiedlichen Rechtsfolgen für die Sammlungsfrist und das Quorum wählen müssen, vgl. Art. 82 Abs. 5 LV TH. 
jeweilige Regierung. ${ }^{89}$ Sieht man in diesem Sinne umfangreiche Sicherungshürden im Vorfeld der Volksabstimmung vor, sind Zustimmungsquoren entbehrlich; sie sind auch deshalb keineswegs zwingend, weil es bei Wahlen (die gemäß Art. 20 Abs. 2 GG gleichrangig neben den Abstimmungen stehen) ebenfalls keine Mindestbeteiligungserfordernisse gibt, um das Wahlergebnis als gültig anzuerkennen. ${ }^{90}$ Es gibt bereits heute einige Bundesländer, die keine Zustimmungsquoren bei Volksentscheiden über (einfache) Gesetze kennen (Bayern, Hessen, Sachsen). ${ }^{91}$

Dasselbe gilt für Volksabstimmungen, die nicht auf Volksbegehren zurückgehen, sondern von den Verfassungsorganen initiiert werden. Denn diese bürgen mit entsprechenden qualifizierten Minderheitsquoren oder Gremienbeschlüssen - ebenso wie hohe Zugangshürden beim Volksbegehren - für den erforderlichen Grad an politischer Bedeutung des Gesetzgebungsvorhabens, um eine ausreichende Repräsentativität sicherzustellen. So verhält es sich auch in dem dieser Untersuchung zugrunde liegenden Fall in Baden-Württemberg. Um eine Volksabstimmung gemäß Art. 60 Abs. 3 LV BW zu initiieren, bedarf es zum einen eines entsprechenden Antrags, hinter dem mindestens ein Drittel der gewählten Landtagsabgeordneten steht, sowie zum anderen einer entsprechenden positiven Beschlussfassung der Landesregierung, die insoweit ein freies politisches Ermessen hat. ${ }^{92}$ Nur wenn beide Organe in diesem Sinne kumulativ zusammenwirken, wird die Volksabstimmung anberaumt. Insofern ist bei diesem Verfahrensweg eine doppelte und damit ausreichende Zugangshürde vorgesehen; lediglich die obligatorische verfassungsgerichtliche Überprüfung (die bei dem hier in Rede stehenden Fall die Volksabstimmung eventuell wegen Verfassungswidrigkeit des Gesetzentwurfs - siehe oben - verhindert hätte) könnte noch ergänzt werden. Eines zusätzlichen Zustimmungsquorums, wie es Art. 60 Abs. 5 Satz 2 LV BW vorschreibt, bedürfte es hier folglich nicht.

\subsection{Rechtsschutzproblem}

Wie bei Wahlen hat auch bei Volksabstimmungen jeder Stimmbürger die Möglichkeit, rechtliche Mängel bei der Durchführung der Abstimmung zu rügen. So sieht in BadenWürttemberg $\$ 21$ Abs. 2 des Volksabstimmungsgesetzes (VAbstG BW) ein ausdrückliches Einspruchsrecht aller Stimmberechtigten vor, mit dem die (erfolgte) Volksabstimmung angefochten werden kann. Gegenstand der Anfechtung kann aber keine (abstrakte) Normen-

89 Siehe zum Beispiel Art. 48 Abs. 2 LV NW; Art. 99 Abs. 3 LV SL; Art. 71 Abs. 2 Satz 2, 3 LV SN; Art. 81 Abs. 2 Satz 1 LV ST; Art. 42 Abs. 1 Satz 3, 4 LV SH; Art. 82 Abs. 3 Satz 3 LV TH.

90 Vgl. Otmar Jung, Zur Problematik des Beteiligungsquorums, in: Lars P. Feld / Peter M. Huber / ders. / Christian Welzel / Fabian Wittreck (Hrsg.), Jahrbuch für direkte Demokratie 2009, BadenBaden 2010, S. 40 - 65, spricht insofern von einer Durchbrechung eines ,erzdemokratischen Prinzips“. Noch weitergehender Fabian Wittreck, a.a.O. (Fn. 38), S. 224 f., der bei einem nur schwer erreichbaren Zustimmungsquorum ,wahrscheinlich“ auch das Homogenitätsgebot des Art. 28 Abs. 1 Satz 1 GG verletzt sieht, da Art. 20 Abs. 2 Satz 2 GG die Gleichrangigkeit von Wahlen und Abstimmungen statuiert.

91 Vgl. Art. 124 Abs. 3 S. 2 LV HE; Art. 72 Abs. 4 LV SN. Dasselbe gilt in Staaten mit besonderer Partizipationskultur wie die Schweiz oder die US-Bundesstaaten, vgl. Otmar Jung, a.a.O. (Fn. 90), S. 40.

92 Vgl. Paul Feuchte, a.a.O. (Fn. 24), Art. 60, Rn. 5. 
kontrolle des der Abstimmung zugrunde liegenden Gesetzentwurfs sein. $\$ 21$ Abs. 4 VAbstG BW beschränkt vielmehr die zulässigen Anfechtungsgründe auf potenziell ergebnisrelevante Verstöße gegen das auf Verfahrensfragen beschränkte VAbstG (und der Stimmordnung) bei der „Vorbereitung oder Durchführung der Volksabstimmung“.

Erst recht gibt es (jedenfalls in Baden-Württemberg) keine Regelung, mit der ein einzelner Stimmbürger bereits im Vorfeld gegen eine geplante Volksabstimmung - etwa wegen Verfassungswidrigkeit des zur Abstimmung kommenden Gesetzentwurfs, aber auch wegen Fehlens der Voraussetzungen für eine Volksabstimmung gemäß Art. 60 LV BW - vorgehen könnte. Folglich kann sich kein Stimmbürger gegen die Heranziehung zu einer formell und/ oder materiell verfassungswidrigen Volksgesetzgebung gerichtlich zur Wehr setzen, was nicht zuletzt vor dem Hintergrund der bürgerschaftlichen Abstimmungspflicht in Art. 26 Abs. 3 LV BW erstaunt und mit Blick auf die Rechtsschutzgarantie des Art. 19 Abs. 4 GG erheblichen verfassungsrechtlichen Bedenken begegnet. Manfred Löwisch hat daher seinen im Vorfeld der Volksabstimmung beim StGH BW gestellten Antrag damit begründet, dass er als Stimmbürger Teil des Stimmorgans Volk und dadurch „für die Volksabstimmung organmäßig teilzuständig sei“, woraus ein eigenständiges verfassungsrechtliches Teilhaberecht folge. Dies würde bedeuten, dass der einzelne Stimmbürger ein Organstreitverfahren gemäß Art. 68 Abs. 1 Satz 1 Nr. 1 LV BW anstrengen könnte. ${ }^{93}$ Dem steht jedoch entgegen, dass - trotz des grundsätzlichen Organcharakters des Volkes (siehe oben) - dem einzelnen Bürger in der Verfassungsrechtsprechung keine (Teil-)Organstellung im Sinne des Organstreitrechts zuerkannt wird $^{94}$; vielmehr steht ihm (nur) in Gestalt der Verfassungsbeschwerde ein entsprechendes Rechtsmittel zu. So hat das BVerfG in seiner Lissabon-Entscheidung aus Art. 146 in Verbindung mit Art. 38 Abs. 1 Satz 1 GG ein (grundrechtsgleiches) Teilhaberecht jedes wahlberechtigten Bürgers auf Mitwirkung an einer neuen Verfassung abgeleitet, das nur mit dem Individual-Instrument der Verfassungsbeschwerde prozessual geltend gemacht werden kann. ${ }^{95}$ Das baden-württembergische Landes(verfassungs)recht kennt aber bislang keine Individual-Verfassungsbeschwerde, weshalb eine Rechtsschutzlücke vorliegt. ${ }^{96}$ Da die Heranziehung eines Stimmbürgers zu einer Abstimmung über einen verfassungswidrigen Gesetzentwurf diesen in seinen grundrechtsähnlichen Rechten gemäß Art. 38 Abs. 1 Satz 1 GG tangiert, bedeutet hier die Nichterfüllung der „allgemeinen staatlichen Justizgewährungspflicht" einen Eingriff in die Rechtsschutzgarantie des Art. 19 Abs. 4 GG. ${ }^{97}$ Mangels Gesetzesvorbehalts sind derartige Eingriffe nur durch andere Grundrechte oder Rechtsgüter von Verfassungsrang zu rechtfertigen ${ }^{98}$, wofür hier keine Anhaltspunkte ersichtlich sind. Daher erscheint es verfassungsrechtlich dringend geboten, für die Heranziehung als Stimmbürger einen entsprechenden Individual-Rechtsbehelf zu schaffen. Dies gilt jedenfalls solan-

93 Vgl. Manfred Löwisch, a.a.O. (Fn. 6), S. 7 f.

94 Vgl. StGH BW, Beschluss vom 17. Oktober 2011 (GR 5/11), S. 2 f. mit weiteren Nachweisen, http://www.baden-wuerttemberg.de/sixcms/media.php/602/Beschlussausfertigung_anonym.pdf (Abruf am 16. März 2012).

95 Vgl. BVerfGE, Bd. 123, S. 267 ff., Rn. 179 f.

96 Allerdings hat die Landesregierung von Baden-Württemberg am 17. April 2012 einen Gesetzentwurf zur Einführung dieses Rechtsbehelfs beschlossen. Vgl. http://www.justiz-bw.de/servlet/PB/ menu/1276134/index.html?ROOT=1153239 (Abruf am 3. Juni 2012).

97 Vgl. Walter Krebs, a.a.O. (Fn. 30), Art. 19, Rn. 55 f.; Bodo Pieroth / Bernhard Schlink, Grundrechte. Staatsrecht II, Heidelberg 2011, Rn. 1113.

98 Vgl. Bodo Pieroth / Bernhard Schlink, a.a.O. (Fn. 97), Rn. 1115. 
ge, bis es - wie oben als zusätzliche Zugangshürde vorgeschlagen - eine verfahrensimmanente verfassungsgerichtliche Prüfung von Volksabstimmungen einschließlich der ihnen zugrunde liegenden Gesetzentwürfe de constitutione ferenda gibt.

Ein interessantes Vorbild ist in diesem Zusammenhang die im Partizipationsrecht häufig erwähnte Schweiz: Dort sieht Art. 85 lit. a des Bundesgesetzes über die Organisation der Rechtspflege (OG) den Individualrechtsbehelf einer so genannten „Stimmrechtsbeschwerde“ vor, wonach gemäß Art. 88 OG jeder Stimmberechtigte (im Vorfeld einer Abstimmung) eine Verletzung der „politischen Stimmberechtigung der Bürger“ auf kantonaler oder kommunaler Ebene gerichtlich geltend machen kann. ${ }^{99}$ Dabei ist zwar zunächst vor allem an Beeinträchtigungen des Wahl- oder Stimmrechts gedacht, aber das Schweizer Bundesgericht hat mittlerweile ausdrücklich anerkannt, dass auch die Zulässigkeit der Volksabstimmung und die Verfassungsmäßigkeit der zugrunde liegenden Initiative mit der Stimmrechtsbeschwerde überprüft werden können. ${ }^{100}$

\section{Fazit: Zulässige Volksabstimmung über höchst fragliches Kündigungsrecht}

Zusammenfassend ist festzustellen, dass die Volksabstimmung in Baden-Württemberg vom 27. November 2011 alle Verfahrensvoraussetzungen erfüllt hat und somit zulässig war. Der ihr zugrunde liegende Gesetzentwurf ist jedoch auf die Ausübung eines Kündigungsrechts gerichtet, das nicht existiert und dessen Eintritt auch nicht mit einer gewissen Wahrscheinlichkeit erwartet werden konnte. Daher sind Wirkungsperspektive und normativer Gehalt dieses Gesetzentwurfs nur mit Mühe zu bejahen. Ob dies noch den Anforderungen des Rechtsstaatsprinzips an den Formentyp des Gesetzes genügt, erscheint durchaus fraglich.

Zugleich hat die baden-württembergische Volksabstimmung das Problempotenzial eines Zustimmungsquorums (im nicht eingetretenen Fall einer unterhalb des Quorums liegenden Abstimmungsmehrheit für das S 21-Kündigungsgesetz) erahnen lassen. Deshalb sollte stärker auf die Zugangs- als auf die Zustimmungshürden gesetzt werden. In diesem Zusammenhang bedürfte es auch einer verfahrensmäßig integrierten Prüfung der Zulässigkeit der Volksabstimmung und der Verfassungsmäßigkeit des Gesetzentwurfs, die durch das zuständige Verfassungsgericht entweder generell oder zumindest auf ein entsprechendes Rechtsschutzbegehren eines Abstimmungsbürgers vorzunehmen wäre.

99 Nicolas von Arx, Die Stimmrechtsbeschwerde, Genf 1997, S. 6, http://www.c2d.ch/files/Report_ vonArx_Stimmrechtsbeschwerde_1997.pdf (Abruf am 16. März 2012).

100 Vgl. ebenda, S. 8 mit weiteren Nachweisen. 